\title{
Atom-molecule theory of broad Feshbach resonances
}

\author{
G. M. Falco and H. T. C. Stoof \\ Institute for Theoretical Physics, Utrecht University, Leuvenlaan 4, 3584 CE Utrecht, The Netherlands
}

(Received 17 February 2005; published 27 June 2005)

\begin{abstract}
We derive the atom-molecule theory for an atomic gas near a broad Feshbach resonance, where the energy dependence of the atom-molecule coupling becomes crucial for understanding experimental results. We show how our many-body theory incorporates the two-atom physics exactly. In particular, we calculate the magnetic moment of a two-component gas of ${ }^{6} \mathrm{Li}$ atoms for a wide range of magnetic fields near the broad Feshbach resonance at about $834 \mathrm{G}$. We find excellent agreement with the experiment of Jochim et al. [Phys. Rev. Lett. 91, 240402 (2003)].
\end{abstract}

DOI: 10.1103/PhysRevA.71.063614

PACS number(s): 03.75. $-\mathrm{b}, 39.25 .+\mathrm{k}, 67.40 .-\mathrm{w}$

\section{INTRODUCTION}

By sweeping an external magnetic field in the right direction across a Feshbach resonance, it is possible to create ultracold diatomic molecules in an atomic gas [1-5]. The reason for this formation of molecules is that, by changing the magnetic field, the energy of the molecular state that causes the Feshbach resonance can be moved from above to below the threshold of the two-atom continuum [6,7]. In an atomic Fermi gas this experimental control over the location of the molecular energy level offers the exciting possibility to study in detail the crossover between the Bose-Einstein condensation (BEC) of diatomic molecules and the BoseEinstein condensation of atomic Cooper pairs-i.e., the Bardeen-Cooper-Schrieffer (BCS) transition [8-11]. In particular, the very broad resonance of ${ }^{6} \mathrm{Li}$ at about $834 \mathrm{G}$ is for this purpose used by a number of experimental groups [12-16].

The use of this broad Feshbach resonance, however, seriously complicates the theoretical analysis of the experimental results, because for such a broad resonance the atommolecule coupling can no longer be assumed to be independent of the relative energy of the colliding atoms. Physically, this implies that the atom-molecule coupling shows retardation effects in this case. Dealing with these retardation effects requires the development of an effective atom-molecule theory that is suitable for many-body calculations and at the same time incorporates the exact energy dependence of the two-body scattering process. It is the main purpose of this paper to show how we can arrive at such an effective atom-molecule theory by generalizing the quantum field theory developed previously for atomic Bose gases in Refs. $[17,18]$ and extended to atomic Fermi gases in Refs. $[19,20]$.

To achieve this goal we need to determine the energy and lifetime of the Feshbach molecule in the presence of the ultracold atomic gas, i.e., we need to determine the molecular self-energy. We therefore first present a many-body approach that can be used to calculate the self-energy exactly. Having the above-mentioned application in mind, we focus here on a two-component Fermi gas. However, our formalism applies also to an atomic Bose gas or to an atomic BoseFermi mixture. After we have obtained the exact expression for the molecular self-energy in the presence of a medium, we then consider the two-body limit of the theory. The reason for considering this limit is twofold. First, any accurate many-body theory for the BEC-BCS crossover in atomic ${ }^{6} \mathrm{Li}$ must incorporate the two-body physics exactly to be able to make a successful comparison with experiments. Second, in this limit we are already able to compare the theory with experimental data on the magnetic moment of an atomic ${ }^{6} \mathrm{Li}$ gas and with coupled-channels calculations of the molecular binding energy. We find excellent agreement in both cases. We therefore conclude that our quantum field theory gives an accurate account of the retardation effects occurring near a broad Feshbach resonance. The theory can thus also be used to investigate the BEC-BCS crossover phenomenon for a broad Feshbach resonance, where subtle two-body threshold effects and strong-coupling many-body physics merge together.

\section{MOLECULAR SELFENERGY}

From now on we consider an incoherent mixture of fermionic atoms in two different hyperfine states, which we denote by $|\uparrow\rangle$ and $|\downarrow\rangle$. If this two-component Fermi gas is in the vicinity of a Feshbach resonance, the effective quantum field theory of the gas is defined by means of the atommolecule Hamiltonian [18,21,22]

$$
\begin{aligned}
H= & \sum_{\mathbf{k}, \sigma}\left(\epsilon_{\mathbf{k}}-\mu\right) a_{\mathbf{k}, \sigma}^{\dagger} a_{\mathbf{k}, \sigma}+\frac{1}{V} \sum_{\mathbf{k}, \mathbf{k}^{\prime}, \mathbf{q}} V_{\mathrm{bg}}(\mathbf{q}) a_{\mathbf{k}+\mathbf{q}, \uparrow}^{\dagger} a_{\mathbf{k}^{\prime}-\mathbf{q}, \downarrow}^{\dagger} a_{\mathbf{k}^{\prime}, \downarrow} a_{\mathbf{k}, \uparrow} \\
& +\sum_{\mathbf{k}}\left(\frac{\epsilon_{\mathbf{k}}}{2}+\epsilon_{\mathrm{bare}}+\Delta \mu B-2 \mu\right) b_{\mathbf{k}}^{\dagger} b_{\mathbf{k}} \\
& +\frac{g_{\text {bare }}}{\sqrt{V}} \sum_{\mathbf{k}, \mathbf{q}}\left(b_{\mathbf{k}}^{\dagger} a_{\mathbf{k} / 2+\mathbf{q}, \uparrow} a_{\mathbf{k} / 2-\mathbf{q}, \downarrow}+\text { H.c. }\right) .
\end{aligned}
$$

Here, $a_{\mathbf{k}, \sigma}^{\dagger}$ is the creation operator of an atom with momentum $\hbar \mathbf{k}$ and in the hyperfine state $|\sigma\rangle$, and $b_{\mathbf{k}}^{\dagger}$ is the creation operator of a bare molecule, i.e., the bound state in the closed channel of the Feshbach problem when the coupling with the open channel is absent. In addition, $\epsilon_{\mathrm{k}}$ is the kinetic energy of an atom, $\mu$ is the chemical potential, $V_{\mathrm{bg}}$ is the nonresonant or background interaction between the atoms, $g_{\text {bare }}$ is 
a)
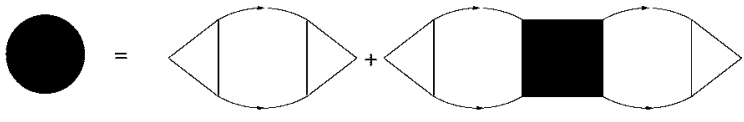

b)

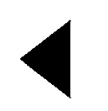

$=1$

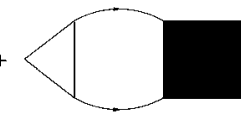

c)
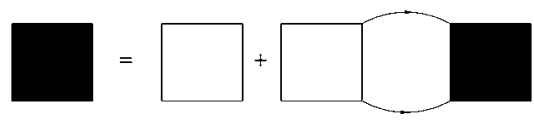

FIG. 1. Diagrammatic representation of (a) the exact molecular self-energy, (b) the renormalization of the atom-molecule coupling, and (c) the Bethe-Salpeter equation for the one-particle irreducible vertex function. Note that triangles denote the atom-molecule coupling, that squares refer to the two- particle vertices, and that the internal lines connecting these quantities represent fully dressed atomic propagators.

the bare or unrenormalized atom-molecule coupling, $B$ is the external magnetic field, $\epsilon_{\mathrm{bare}}$ is the energy of a bare molecule with zero total momentum, and $\Delta \mu$ is the difference in magnetic moment between the bare molecule and two atoms.

Given the above atom-molecule Hamiltonian, the molecular self-energy can, in the normal state of the atomic gas, be calculated from the following procedure. We consider here only the normal state of the atomic gas, because we ultimately want to compare our results with the experiment of Jochim et al. [4]. The generalization to the superfluid state is also of interest, but will be reported elsewhere. We start by expressing the self-energy $\hbar \Sigma_{\mathrm{m}}$ of a single molecule in the atomic gas in terms of the exact atomic Green's functions $G_{\sigma}$ and the exact (four-point) vertex function $\Gamma_{\sigma, \sigma^{\prime}}$ as

$$
\hbar \Sigma_{\mathrm{m}}=-\frac{1}{\hbar} g_{\text {bare }}\left(G_{\uparrow} G_{\downarrow}-\frac{1}{\hbar} G_{\uparrow} G_{\downarrow} \Gamma_{\uparrow, \downarrow} G_{\uparrow} G_{\downarrow}\right) g_{\text {bare }} .
$$

Note that we are using an operator notation here that suppresses the dependence on momenta and Matsubara frequencies of the various quantities involved. The diagrammatic equivalent of Eq. (2) is shown in Fig. 1(a).

This result can be clarified by introducing the exact atommolecule coupling $g$ by means of [see Fig. 1(b)]

$$
g=\left(1-\frac{1}{\hbar} \Gamma_{\uparrow, \downarrow} G_{\uparrow} G_{\downarrow}\right) g_{\text {bare }},
$$

since then the self-energy can be put into the expected form

$$
\hbar \Sigma_{\mathrm{m}}=-\frac{1}{\hbar} g_{\text {bare }} G_{\uparrow} G_{\downarrow} g
$$

Because the exact vertex function is one-particle irreducible, it satisfies the Bethe-Salpeter equation

$$
\Gamma_{\uparrow, \downarrow}=\Gamma_{\uparrow, \downarrow}^{\mathrm{ir}}-\frac{1}{\hbar} \Gamma_{\uparrow, \downarrow}^{\mathrm{ir}} G_{\uparrow} G_{\downarrow} \Gamma_{\uparrow, \downarrow},
$$

whose diagrammatic equivalent is shown in Fig. 1(c). The quantity $\Gamma_{\sigma, \sigma^{\prime}}^{\mathrm{ir}}$ is the exact vertex function that is two-particle irreducible in the particle-particle channel. From Eqs. (4) and (5) it appears that the self-energy depends on the bare atom- molecule vertex. However, the definition of the exact atommolecule coupling can be used to eliminate the bare coupling from the theory.

\section{ATOMIC PHYSICS}

The two-body limit is obtained by replacing in the above procedure the exact atomic Green's functions by the noninteracting atomic Green's functions and the two-particle irreducible vertex function by $V_{\mathrm{bg}}$. Performing the required calculations, we then find that the energy-dependent dressed atom-molecule coupling becomes

$$
g^{2 \mathrm{~B}}(E)=g(B) \frac{1}{1-\left[a_{\mathrm{bg}}(B) / \hbar\right] \sqrt{-m E^{+}}},
$$

where $E^{+}=E+i 0$ and both the background scattering length $a_{\mathrm{bg}}(B)$ of the background interaction $V_{\mathrm{bg}}$ and the zero-energy coupling constant $g(B)$ are known experimental quantities. Moreover, the molecular self-energy obeys

$$
\hbar \Sigma^{2 \mathrm{~B}}(E)-\hbar \Sigma^{2 \mathrm{~B}}(0)=\frac{\eta(B) \sqrt{-E^{+}}}{1+\left|a_{\mathrm{bg}}(B) / \hbar\right| \sqrt{-m E^{+}}},
$$

where the energy $\eta^{2}(B)=g^{4}(B) m^{3} / 16 \pi^{2} \hbar^{6}$ defines an important energy scale in the problem, which is related to the width of the Feshbach resonance.

The energy of the dressed molecular state with zero kinetic energy $\epsilon_{\mathrm{m}}$ is determined by the poles of the full molecular propagator, which is equivalent to solving the equation

$$
\epsilon_{\mathrm{m}}=\delta(B)+\frac{\eta(B) \sqrt{-\epsilon_{\mathrm{m}}^{+}}}{1+\left|a_{\mathrm{bg}}(B) / \hbar\right| \sqrt{-m \epsilon_{\mathrm{m}}^{+}}},
$$

where $\delta(B)=\Delta \mu B+\epsilon_{\text {bare }}+\hbar \Sigma^{2 \mathrm{~B}}(0) \equiv \Delta \mu\left(B-B_{0}\right)$ is known as the detuning and $B_{0}$ is the magnetic field location of the Feshbach resonance. For positive detuning there only exists a solution with a negative imaginary part, in agreement with the fact that the molecule decays when its energy is above the two-atom continuum threshold. For negative detuning the dressed molecular propagator has a real pole at negative energy corresponding to the bound-state energy of the dressed molecule.

Near resonance the bound-state energy $\epsilon_{\mathrm{m}}(B)$ becomes small and we are allowed to put the right-hand side of Eq. (8) equal to zero. In this approximation the location of the pole can be found analytically and yields the expected result

$$
\epsilon_{\mathrm{m}}(B)=-\frac{\hbar^{2}}{m a^{2}(B)},
$$

where the total scattering length $a(B)$ is given by

$$
\frac{4 \pi \hbar^{2} a(B)}{m}=\frac{4 \pi \hbar^{2} a_{\mathrm{bg}}(B)}{m}-\frac{g^{2}(B)}{\delta(B)} .
$$

The general solution, which is required when we want to know the bound-state energy over a large range of magnetic fields, can only be found numerically as we show shortly. The residue of the pole is in general given by 


$$
Z(B)=\left[1-\frac{\partial \hbar \Sigma^{2 \mathrm{~B}}(E)}{\partial E}\right]_{E=\epsilon_{\mathrm{m}}(B)}^{-1},
$$

and it is always smaller than 1 . This is because the dressed molecular state near the Feshbach resonance obeys

$$
\begin{aligned}
\left.\langle\mathbf{r}| \chi_{\mathrm{m}} ; \text { dressed }\right\rangle \simeq & \left.\sqrt{Z(B)} \chi_{\mathrm{m}}(\mathbf{r}) \mid \text { closed }\right\rangle \\
& \left.+\sqrt{1-Z(B)} \frac{1}{\sqrt{2 \pi a(B)}} \frac{e^{-r / a(B)}}{r} \mid \text { open }\right\rangle,
\end{aligned}
$$

where $\chi_{\mathrm{m}}(\mathbf{r})$ denotes the wave function of the bare molecular state in the closed channel of the Feshbach problem. The dressed molecular state therefore only contains with an amplitude $\sqrt{Z(B)}$ the bare molecular state $\mid \chi_{\mathrm{m}}$; closed $\rangle$ of the closed channel.

\section{MAGNETIC MOMENT}

Since the open and closed channels of the Feshbach resonance in atomic ${ }^{6} \mathrm{Li}$ experiments correspond in an excellent approximation to the electronic triplet and singlet states, respectively, we have $\Delta \mu=2 \mu_{B}$, with $\mu_{B}$ the Bohr magneton. As a result, the magnetic moment of the dressed molecules $\mu_{\mathrm{m}}$ as a function of magnetic field is equal to

$$
\mu_{\mathrm{m}}(B)=2 \mu_{B}[1-Z(B)] .
$$

Close to resonance where $Z(B) \ll 1$ and the contribution of the open channel becomes large, the magnetic moment approaches the value $2 \mu_{B}$ characteristic of the triplet state. Far off resonance we have $Z(B) \simeq 1$ and the dressed state is almost equal to the bound state of the closed-channel potential whose electronic spin is a singlet. In that case we thus have that $\mu_{\mathrm{m}}(B)$ goes to zero.

The magnetic moment of the molecules obtained with the very broad Feshbach resonance at about $834 \mathrm{G}$ in a gas of ${ }^{6} \mathrm{Li}$ atoms has been measured by Jochim et al. [4]. For such a broad resonance, the background scattering length $a_{\mathrm{bg}}(B)$ and the zero-energy atom-molecule coupling constant $g(B)$, which, together with the magnetic field $B_{0}$, constitute the only input parameters of our theory, depend strongly on the magnetic field over the relevant experimental range. In contrast to narrow resonances, neglecting these magnetic field dependences is not sufficiently accurate here. Therefore, we use the field dependence of $a_{\mathrm{bg}}(B)$ that was calculated by von Kempen et al. from a careful analysis of the experimental knowledge of the singlet and triplet interatomic potentials [23]. The field-dependent atom-molecule coupling constant is deduced from $a_{\mathrm{bg}}(B)$ by using Eq. (10) and the known total scattering length $a(B)$ [24]. Nevertheless, it is worth pointing out that our results do not depend sensitively on the particular input used for $a_{\mathrm{bg}}(B)$. We have checked explicitly that other choices of $a_{\mathrm{bg}}(B)$, which are compatible with the general constraints on the background scattering length imposed by the atomic physics outside the Feshbach resonance $[23,24]$, lead to the same results.

In Fig. 2 we show the comparison between the experimental data and the theoretical result from Eq. (12), after solving numerically Eq. (8) for the molecular bound-state

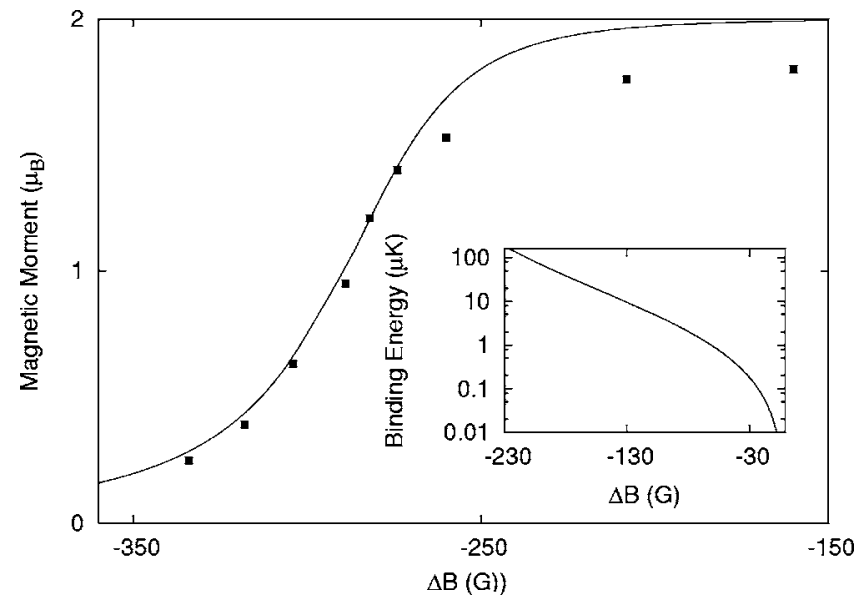

FIG. 2. Magnetic moment of the dressed molecules as a function of the external magnetic field $\Delta B=\left(B-B_{0}\right)$. The dots represent the experimental data of Ref. [4], while the solid line is our theoretical result. For the field-dependent background scattering length $a_{\mathrm{bg}}(B)$ we have used the parametric expression obtained in Ref. [23]. The inset also shows the molecular two-body bound-state energy as a function of magnetic field, which shows excellent agreement with the coupled-channels calculation given in Ref. [4].

energy and substituting this into Eq. (11). It is important to consider the full energy dependences in Eq. (7) to obtain excellent agreement with experiment. Neglecting the energy corrections in the denominator of the self-energy, the curve for the magnetic moment would drop much slower to zero such that, in the range considered in Fig. 2, it would essentially be equal to the horizontal line $\mu_{\mathrm{m}}(B)=2 \mu_{B}$. Due to the large values of the background scattering length, also an expansion of the self-energy for small energies in the spirit of Fermi liquid theory [25], i.e.,

$$
\begin{aligned}
& \hbar \Sigma^{2 \mathrm{~B}}(E)-\hbar \Sigma^{2 \mathrm{~B}}(0) \\
& \quad \simeq \eta(B) \sqrt{-m E^{+}} \times\left[1-\left|a_{\mathrm{bg}}(B) / \hbar\right| \sqrt{-m E^{+}}\right],
\end{aligned}
$$

is a good approximation only very close to resonance when $|\Delta B|<100 \mathrm{G}$. At larger negative detunings, over the range of the magnetic field where the experimental points are taken, the expansion breaks down and leads to unphysical results.

Our theoretical curve exhibits close to resonance the same quantitative deviation from the experiment as the coupledchannels calculation given in Ref. [4]. Jochim et al. [4] attribute the origin of this systematic deviation to the fact that in principle the atoms and molecules experience a different optical trapping potential. We believe, however, that this explanation is unlikely because the deviation between the theoretical and experimental results occurs close to resonance where $Z(B) \ll 1$ and the dressed-molecule wave function consists almost completely of atoms in the open channel. As an alternative explanation we suggest that many-body effects could be important in order to describe the reduction of the molecular magnetic moment. Such many-body corrections are expected to become important when the gas parameter $k_{F} a(B)$ is no longer small, where $k_{F}$ refers to the Fermi momentum of the gas. For the densities used in Ref. [4] it turns 
out that in the range where the deviation occurs we have that $k_{F} a(B) \geqslant 0.1$. In contrast to coupled-channels calculations, many-body corrections to the molecular magnetic moment are easily incorporated in our quantum field theory approach. They are simply determined by Eqs. (2)-(5) and are an important topic of further investigations.

\section{CONCLUSIONS}

In this paper we have developed a many-body atommolecule theory for broad Feshbach resonances where retardation effects in the atom-molecule coupling cannot be neglected. The theory is shown to reproduce the two-body physics exactly. As an application, we have calculated the magnetic moment of an atomic ${ }^{6} \mathrm{Li}$ gas and the molecular binding energy. The agreement with experimental data and with coupled-channels calculations is excellent. We believe, therefore, that the results obtained in this paper constitute a minimal input for every theory that aims at a complete understanding of the BCS-BEC crossover physics in atomic ${ }^{6} \mathrm{Li}$ near the broad Feshbach resonance at about $834 \mathrm{G}$. Work in this latter direction is presently being completed.

\section{ACKNOWLEDGMENTS}

We thank Randy Hulet, Servaas Kokkelmans, and Bout Marcelis for very stimulating discussions. This work is supported by the Stichting voor Fundamenteel Onderzoek der Materie (FOM) and the Nederlandse Organisatie voor Wetenschaplijk Onderzoek (NWO).
[1] M. Greiner, C. A. Regal, and D. S. Jin, Nature (London) 426, 537 (2003).

[2] K. E. Strecker, G. B. Partridge, and R. G. Hulet, Phys. Rev. Lett. 91, 080406 (2003).

[3] J. Cubizolles, T. Bourdel, S. J. J. M. F. Kokkelmans, G. V. Shlyapnikov, and C. Salomon, Phys. Rev. Lett. 91, 240401 (2003).

[4] S. Jochim, M. Bartenstein, A. Altmeyer, G. Hendl, C. Chin, J. H. Denschlag, and R. Grimm, Phys. Rev. Lett. 91, 240402 (2003).

[5] M. W. Zwierlein, C. A. Stan, C. H. Schunck, S. M. F. Raupach, S. Gupta, Z. Hadzibabic, and W. Ketterle, Phys. Rev. Lett. 91, 250401 (2003).

[6] W. C. Stwalley, Phys. Rev. Lett. 37, 1628 (1976).

[7] E. Tiesinga, B. J. Verhaar, and H. T. C. Stoof, Phys. Rev. A 47, 4114 (1993).

[8] H. T. C. Stoof, M. Houbiers, C. A. Sackett, and R. G. Hulet, Phys. Rev. Lett. 76, 10 (1996).

[9] E. Timmermans, K. Furuya, P. W. Milonni, and A. K. Kerman, Phys. Lett. A 285, 228 (2001).

[10] Y. Ohashi and A. Griffin, Phys. Rev. Lett. 89, 130402 (2002); Phys. Rev. A 67, 033603 (2003); 67, 063612 (2003).

[11] J. N. Milstein, S. J. J. M. F. Kokkelmans, and M. J. Holland, Phys. Rev. A 66, 043604 (2002).

[12] M. Bartenstein, A. Altmeyer, S. Riedl, S. Jochim, C. Chin, J. H. Denschlag, and R. Grimm, Phys. Rev. Lett. 92, 120401 (2004).

[13] M. W. Zwierlein, C. A. Stan, C. H. Schunck, S. M. F. Raupach,
A. J. Kerman, and W. Ketterle, Phys. Rev. Lett. 92, 120403 (2004).

[14] J. Kinast, S. L. Hemmer, M. E. Gehm, A. Turlapov, and J. E. Thomas, Phys. Rev. Lett. 92, 150402 (2004).

[15] T. Bourdel, L. Khaykovich, J. Cubizolles, J. Zhang, F. Chevy, M. Teichmann, L. Tarruell, S. J. J. M. F. Kokkelmans, and C. Salomon, Phys. Rev. Lett. 93, 050401 (2004).

[16] K. E. Strecker, G. B. Partridge, R. I. Kamar, and R. G. Hulet, in Proceedings of the 19th International Conference on Atomic Physics (AIP, Melville, NY, in press).

[17] R. A. Duine and H. T. C. Stoof, J. Opt. B: Quantum Semiclassical Opt. 5, S212 (2003).

[18] R. A. Duine and H. T. C. Stoof, Phys. Rep. 396, 115 (2004).

[19] G. M. Falco, R. A. Duine, and H. T. C. Stoof, Phys. Rev. Lett. 92, 140402 (2004).

[20] G. M. Falco and H. T. C. Stoof, Phys. Rev. Lett. 92, 130401 (2004).

[21] K. V. Kheruntsyan and P. D. Drummond, Phys. Rev. A 61, 063816 (2000).

[22] E. Timmermans, P. Tommasini, M. Hussein, and A. Kerman, Phys. Rep. 315, 199 (1999).

[23] E. G. M. von Kempen, B. Marcelis, and S. J. J. M. F. Kokkelmans, e-print cond-mat/0406722.

[24] M. Houbiers, H. T. C. Stoof, W. I. McAlexander, and R. G. Hulet, Phys. Rev. A 57, R1497 (1998).

[25] G. M. Bruun and C. J. Pethick, Phys. Rev. Lett. 92, 140404 (2004). 